\title{
Profil épidémiologique d'une intoxication au méthanol, El Hajeb (Maroc)
}

Sanah Essayagh, ${ }^{1}$ Mariama Bahalou, ${ }^{2 *}$ Meriem Essayagh ${ }^{3}$ et Touria Essayagh ${ }^{4 *}$

${ }^{1}$ Laboratoire Agroalimentaire et Santé, Faculté des Sciences et Techniques, Université Hassan ler, Settat (Maroc). ${ }^{2}$ Délégation de la Santé, Meknès (Maroc). ${ }^{3}$ Faculté de Médecine et de Pharmacie, Université Mohammed V, Rabat (Maroc). ${ }^{4}$ Laboratoire Sciences et Technologies de la Santé, Institut Supérieur des Sciences de la Santé, Université Hassan ler, Settat (Maroc) (Correspondance à adresser à : essayagh.toula@gmail.com (TE). *Ces auteurs ont apporté la même contribution au travail.

\section{Résumé}

Contexte : l'intoxication au méthanol est un problème pertinent dans les pays en développement. Nous signalons une intoxication au méthanol qui s'est produite le 22 mai 2017 à El Hajeb (Maroc).

Objectifs : décrire l'ampleur de l'intoxication, déterminer sa source et instaurer les mesures préventives nécessaires.

Méthodes : nous avons mené une enquête transversale. Un questionnaire normalisé comprenant des données socio-économiques, les symptômes cliniques et l'heure de consommation a été administré en face à face aux cas. Des prélèvements biologiques ont été effectués pour analyse toxicologique et physico-chimique. Les données ont été saisies et analysées sur Epi Info version 7.

Résultats : au total, 26 cas ont été colligés avec un âge moyen de 39,7 (écart type [ET] 11,1) ans et un sex ratio homme/ femme de 5,5. Tous les cas intoxiqués étaient de faible niveau socio-économique. La durée moyenne de latence entre consommation et apparition des symptômes était de 1,5 (ET 1) jours. Les symptômes signalés étaient les faibles troubles de la conscience chez 14 cas (53,8\%), les douleurs abdominales chez 10 cas (38,5\%), les céphalées chez neuf cas (34,6 \%), les vomissements chez huit cas (30,8\%) et le coma chez sept cas (27,1\%). La létalité a été de $65 \%$ et quatre cas ont développé une cécité. Les résultats de laboratoire ont confirmé la présence de méthanol dans le sang avec des valeurs supérieures à 0,6 g/L. La dose de méthanol dans la bouteille incriminée était de $217 \mathrm{~g} / \mathrm{L}$.

Conclusion : la sensibilisation de la population au danger du méthanol est importante. Une sensibilisation des professionnels de santé aux signes cliniques et à la conduite à tenir face à une intoxication au méthanol est nécessaire.

Citation: Essayagh S; Bahalou M; Essayagh M; Essayagh T. Profil épidémiologique d'une intoxication au méthanol, El Hajeb (Maroc). East Mediterr Health J. 2020;26(11):1425-1429. https://doi.org/10.26719/2020.26.11.1425

Mots-clés : méthanol, intoxication, El Hajeb

Reçu : 01/10/19; accepté : 16/12/19

(c) Organisation mondiale de la Santé 2020 Certains droits réservés. La présente publication est disponible sous la licence Creative Commons Attribution - Pas d'utilisation commerciale - Partage dans les mêmes conditions 3.0 IGO licence (CC BY-NC-SA 3.0 IGO; https://creativecommons. org/licenses/by-nc-sa/3.0/igo ).

\section{Introduction}

L'alcool à brûler contient des concentrations variables de méthanol. Ce dernier est utilisé dans les antigels des automobiles et les solvants (1). Il est aussi utilisé comme substitut de l'alcool éthylique dans plusieurs boissons alcoolisées frelatées dans la classe de faible niveau socioéconomique dans les pays en développement, surtout en cas de prohibition de l'alcool. Le méthanol peut être ingéré de manière accidentelle ou avec intention suicidaire, entraînant ainsi des intoxications sous forme isolée ou collective. À l'intérieur du corps, il est métabolisé en acide formique qui, sans traitement, entraîne la cécité ou la mort. Les études montrent des létalités allant de $76 \%$ à $89 \%(2,3)$. Le méthanol peut également affecter le tissu cérébral. L'évolution de l'intoxication au méthanol est conditionnée par le degré d'acidose et le délai entre l'exposition et l'instauration du traitement spécifique (1). Les signes cliniques de l'intoxication au méthanol sont non spécifiques, aboutissant à un diagnostic et un traitement tardifs, et à une surmortalité. Le traitement se base soit sur l'administration d'éthanol pour obtenir une concentration constante d'éthanol d'environ $\lg / \mathrm{L}(4,5)$, soit sur l'hémodialyse pour épuration $(5,6)$.
Dans le cadre d'une alerte, l'équipe de surveillance épidémiologique d'El Hajeb a informé le 22 mai 2017, à 10 h 30, l'équipe de Meknès du transfert aux urgences du Centre hospitalier préfectoral ( $\mathrm{CHP}$ ) de Meknès de quatre cas groupés présentant des troubles visuels, des douleurs abdominales et des vomissements. Une enquête épidémiologique pour vérification des cas a été effectuée afin de confirmer l'existence d'une intoxication collective, de décrire son ampleur, de déterminer sa source et d'instaurer les mesures préventives.

\section{Méthodes}

\section{Site de l'enquête}

Pays d'Afrique du nord, le Maroc a une population de 33,8 millions d'habitants. Il se compose de 12 régions, dont celle de Fès-Meknès. Cette région est composée de neuf provinces, dont El Hajeb, qui regroupe 16 communes et compte une population d'environ 35282 habitants (7). Elle dispose d'un hôpital provincial qui, en présence de cas sévères, transfère les patients à l'hôpital provincial de Meknès ou au centre hospitalier régional (CHU) de Fès. 


\section{Schéma d'étude et définition de cas}

Nous avons mené une enquête transversale à visée descriptive. L'enquête s'est déroulée durant la période allant 22 au 29 mai 2017. Elle a concerné MeknèsEl Hajeb-Fès. Un cas a été défini comme toute personne habitant El Hajeb, ayant consommé une boisson alcoolisée et ayant présenté, entre le 17 et le 23 mai 2017, au moins l'un des signes suivants : troubles visuels, céphalées, vomissements, nausées, douleurs abdominales, altération de l'état général, troubles de la conscience ou coma. Les cas ont été recensés à partir du registre des urgences de l'hôpital d'El Hajeb. Une recherche active des cas a aussi été menée auprès de la population d'El Hajeb, en concertation avec les autorités locales. Un interrogatoire avec les malades survivants ou leurs proches a été réalisé. Le suivi des cas a été effectué auprès des hôpitaux d'El Hajeb et de Meknès, et des services de réanimation et d'ophtalmologie du CHU de Fès.

\section{Épidémiologie descriptive}

Un questionnaire a été administré en face à face pour recueillir des informations sur : 1 ) les données sociodémographiques ; 2 ) les signes cliniques ; 3 ) la date de consommation de méthanol et d'apparition des symptômes; 4) le lieu de procuration de l'alcool ; 5) l'évolution du malade; et 6) les complications. Le fournisseur agréé et les cas ont été interrogés sur la procédure de préparation du méthanol. La police judiciaire d'El Hajeb a procédé à la saisie du lot de méthanol pour stopper l'épidémie et assurer l'analyse toxicologique et physico-chimique. Les données ont été analysées sur Epi Info version 7.

\section{Enquête toxicologique et physico-chimique}

Des prélèvements biologiques ont été effectués et envoyés pour analyse au Centre Antipoison et de Pharmacovigilance. Le méthanol et l'éthanol ont été dosés par chromatographie en phase gazeuse (gas chromatography, GC) avec un détecteur à ionisation de flamme (flame ionisation detector, FID) couplée à un échantillonneur automatique d'extraction en espace de tête (headspace, HS) (GC-FID-HS). La recherche des drogues a été réalisée par des méthodes immunochromatographiques.

\section{Considérations éthiques}

Tous les participants avaient donné leur consentement informel verbal. Les tests de laboratoire ont été réalisés à la suite d'un diagnostic de routine.

\section{Résultats}

\section{Épidémiologie descriptive}

Durant l'épidémie, 26 cas ont été identifiés. L'âge moyen était de 39,7 (ET 11,1) ans, avec des extrêmes allant de 28 à 58 ans et un sex ratio homme/femme de 5,5 . Tous les patients étaient de faible niveau socio-économique, sans domicile fixe pour six cas $(23,1 \%)$ (Tableau 1). La durée

\begin{tabular}{|c|c|}
\hline \multicolumn{2}{|c|}{$\begin{array}{l}\text { Tableau 1 Caractéristiques des cas d'intoxication au } \\
\text { méthanol, El Hajeb (Maroc), mai } 2017\end{array}$} \\
\hline Caractéristiques & $\begin{array}{c}\text { Total } \\
(n=26) \\
\text { Nbre }(\%)\end{array}$ \\
\hline Âge moyen (ans) & 39,7 (ET 1,1) \\
\hline \multicolumn{2}{|l|}{ Sexe } \\
\hline Masculin & $22(84,6)$ \\
\hline Féminin & $4(15,4)$ \\
\hline \multicolumn{2}{|c|}{ Situation matrimoniale } \\
\hline Non marié & $21(80,8)$ \\
\hline Marié & $5(19,2)$ \\
\hline \multicolumn{2}{|l|}{ Revenu mensuel (US\$) } \\
\hline$<150$ & $26(100,0)$ \\
\hline$\geq 150$ & $0(0,0)$ \\
\hline \multicolumn{2}{|l|}{ Type de domicile } \\
\hline Sans domicile fixe & $6(23,1)$ \\
\hline Avec domicile fixe & $20(76,9)$ \\
\hline \multicolumn{2}{|c|}{ Consommation d'alcool local } \\
\hline Oui & $20(76,9)$ \\
\hline Non & $6(23,1)$ \\
\hline \multicolumn{2}{|l|}{ Toxicomanie } \\
\hline Oui & $18(69,2)$ \\
\hline Non & $8(30,8)$ \\
\hline
\end{tabular}

moyenne de latence entre consommation et apparition des symptômes était de 1,5 (ET 1) jour. Les cas ont commencé à se produire le 20 mai pour atteindre le maximum le 21 mai avec 11 cas. Le dernier cas a été enregistré le 23 mai à 18 heures (Figure 1). Les symptômes les plus enregistrés étaient les suivants : faibles troubles de la conscience chez 14 cas $(53,8 \%)$, douleurs abdominales chez 10 cas $(38,5 \%)$, céphalées chez neuf cas (34,6\%), vomissements chez huit cas $(30,8 \%)$ et coma chez sept cas (27,1\%). Dix-sept personnes sont décédées, soit une létalité de $65 \%$, et quatre

Figure 1 Courbe épidémique de l'intoxication au méthanol, El Hajeb (Maroc), 22 mai 2017

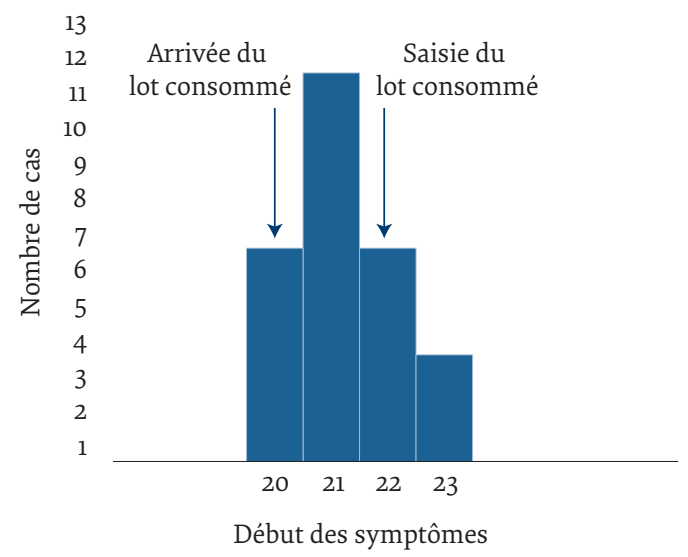


ont développé une cécité, soit 15,4\% (Tableau 2). Vingt cas ont déclaré avoir consommé de l'alcool frelaté (alcool préparé localement), qui provenait du même fournisseur agréé d'El Hajeb.

\section{Enquête toxicologique et physico-chimique}

Les résultats des trois prélèvements biologiques des patients ont révélé la présence de méthanol dans le sang avec des valeurs de 0,7 g/L, 1,35 g/L et 1,94 g/L. Le niveau d'éthanol dans le sang était inférieur à $0,1 \mathrm{~g} / \mathrm{L}$. Les prélèvements d'urine ont révélé la consommation de cannabis chez un malade $(59 \mathrm{mg} / \mathrm{mL})$. Les flacons d'alcool toxique saisis par la police ont montré des concentrations de méthanol de $217 \mathrm{~g} / \mathrm{L}, 7 \mathrm{~g} / \mathrm{L}$ et $6 \mathrm{~g} / \mathrm{L}$.

\section{Discussion}

L'intoxication au méthanol est un problème dans les pays en développement. Au Maroc, une intoxication au méthanol a eu lieu en 1996 avec 76 cas, dont sept décès et quatre cas de cécité (8-11). La majorité des cas d'intoxication au méthanol résultent d'une ingestion/ inhalation ou d'une absorption transcutanée (12). La dose létale est de $1 \mathrm{~mL} / \mathrm{kg}$ (13). La dose toxique de méthanol dépend de l'individu et du traitement reçu. Une concentration de méthanol dans le sang supérieure à $500 \mathrm{mg} / \mathrm{L}$ est associée à des toxicités graves alors qu'une concentration supérieure à 1500-2000 mg/L entraîne la mort chez les patients non traités (14).

Dans notre étude, les trois patients pour lesquels des prélèvements biologiques ont pu être effectués et chez qui les valeurs du méthanol dans le sang étaient de $0,7 \mathrm{~g} / \mathrm{L}$, $1,35 \mathrm{~g} / \mathrm{L}$ et 1,94 g/L sont décédés. Ceci pourrait expliquer la létalité élevée dans notre étude. Dans l'enquête, tous les cas étaient de faible niveau socio-économique, ce qui est compatible avec la littérature (15). Dans notre étude, on a constaté une prédominance masculine. Ceci pourrait être expliqué par le contexte marocain où seule une minorité de femmes sont consommatrices de méthanol, ce qui est semblable à l'étude de Sanaei-Zadeh (16).

Les signes cliniques et la consommation d'alcool à brûler suggèrent une intoxication au méthanol. Cela a été confirmé lors de l'analyse des lots d'alcool frelaté. La répartition des cas par moment d'apparition des symptômes suggère une source commune ponctuelle d'intoxication. En effet, lors de l'enquête, le lot de méthanol incriminé a été celui du 19 mai. Le cas index a été un consommateur du 20 mai. Ce dernier avait présenté une douleur abdominale vers 20 heures, pour laquelle il a été traité. Le 21 mai, à 2 h 30, il est retourné à l'hôpital pour aggravation de cas, où il décède. La létalité de $65 \%$ dans notre série est proche des valeurs observées dans la littérature qui oscillent entre $76 \%$ et $89 \%(2,3)$. La littérature montre que, lors de l'ingestion de méthanol, l'intoxication passe par plusieurs phases : la phase de

\begin{tabular}{lc}
\hline $\begin{array}{l}\text { Tableau } 2 \text { Répartition des symptômes de l'intoxication au } \\
\text { méthanol, El Hajeb (Maroc), mai } 2017\end{array}$ & $\begin{array}{c}\text { Fréquence (\%) } \\
\text { (n = 26) }\end{array}$ \\
\hline Symptômes & $14(53,8)$ \\
\hline Faibles troubles de la conscience & $10(38,5)$ \\
Douleurs abdominales & $9(34,6)$ \\
Céphalées & $8(30,8)$ \\
Vomissements & $7(27,1)$ \\
Coma & $6(23,1)$ \\
Polypnée & $5(19,2)$ \\
Troubles visuels & $4(15,4)$ \\
Cécité & $4(15,4)$ \\
Irritabilité &
\end{tabular}

latence comprise entre 9-24 heures en moyenne et liée à la dose ingérée ; la phase de symptomatologie initiale de l'intoxication qui est peu spécifique et qui est marquée par des troubles neurologiques, des signes digestifs et une polyurie associée à une polydipsie; et la phase d'état où le méthanol ingéré est oxydé au niveau hépatique en formaldéhyde et en acide formique par des enzymes. Laccumulation de l'acide formique est responsable de la toxicité et sa concentration est en lien avec la morbidité, la mortalité et la profondeur de l'acidose métabolique observée dans l'intoxication au méthanol (17). Cet intervalle libre de plusieurs heures entre l'ingestion du méthanol et l'apparition des signes cliniques explique le retard dans la prise en charge des cas et la létalité élevée. À cela pourrait s'ajouter le manque de connaissances de la part des professionnels de santé sur la conduite à tenir face à une intoxication au méthanol.

\section{Limites de l'étude}

Notre étude a connu certaines limites, à savoir, la non-réalisation des prélèvements biologiques pour l'ensemble des patients, d'où la non-collecte des données en relation avec la dose de méthanol dans le sang, et la non-collecte des données sur la quantité d'alcool ingérée.

\section{Conclusion}

L'enquête a révélé une intoxication collective au méthanol. Une sensibilisation du grand public au danger du méthanol est importante. Une sensibilisation des professionnels de santé aux signes cliniques et à la conduite à tenir face à une intoxication au méthanol s'avère nécessaire.

Financement : aucun.

Conflits d'intérêts : aucun déclaré. 


\title{
Epidemiological profile of methanol poisoning, El Hajeb, Morocco
}

\begin{abstract}
Background: Methanol poisoning is of particular importance in low and middle-income countries. We reported on a methanol poisoning incident that occurred 22 May 2017, in El Hajeb (Morocco).
\end{abstract}

Aims: This study aimed to describe the extent of the intoxication, determine its source and implement the necessary preventative measures.

Methods: We conducted a cross-sectional survey. A standardized questionnaire including socio-economic data, clinical symptoms and time of use was administered face-to-face to cases of methanol poisoning. Biological samples were taken for toxicological analysis. Data were entered and analyzed on Epi Info version 7.

Results: Twenty-six cases of methanol poisoning were surveyed with a mean age of 39.7 (SD 11.1) years and a male/female sex ratio of 5.5. All intoxicated cases were of low socioeconomic status. The mean latency period between use and symptom onset was 1.5 (SD 1) days. Reported symptoms were mildly altered consciousness in 14 cases (53.8\%), abdominal pain in 10 cases (38.5\%), headache in 9 cases (34.6\%), vomiting in 8 cases (30.8\%) and coma in 7 cases (27.1\%). Mortality was $65 \%$ and 4 cases developed blindness. Laboratory results confirmed the presence of methanol in the blood with values greater than $0.6 \mathrm{~g} / \mathrm{L}$. The dose of methanol in the associated bottle was $217 \mathrm{~g} / \mathrm{L}$.

Conclusion: Public awareness of the dangers of methanol intoxication is important. Health professionals need to be aware of the clinical signs and what to do in the event of methanol poisoning.

$$
\begin{aligned}
& \text { المرتسم الوبائي للتسمم بالميثانول، مدينة الحاجب، المغرب } \\
& \text { سنا الصايغ، مريمة بحالو، مريم الصايخ، تورية الصايغ }
\end{aligned}
$$

الخلفية: يمثل التسمه بالميثانول مشكلةً خاصة في البلدان المنخفضة والمتو سطة الدخل. وقد أبلغنا عن حالة تسمم بالميثانول حدثت بتاريخ 22 مايو / أيار 2017 في مدينة الحاجب المغربية.

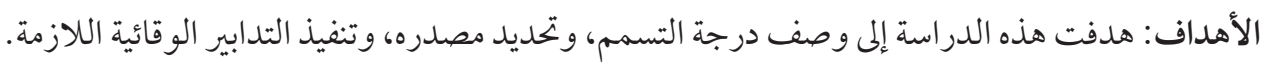

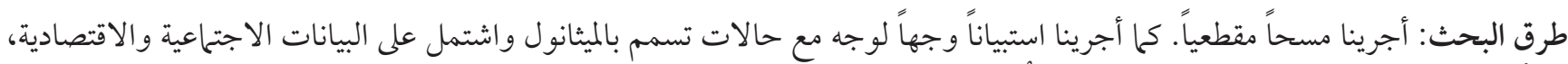

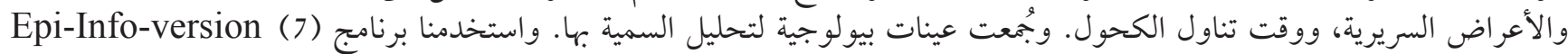

$$
\text { ل إإدخال البيانات وتحليلها. }
$$

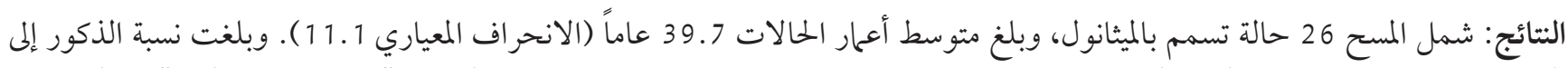

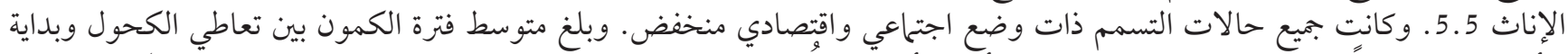

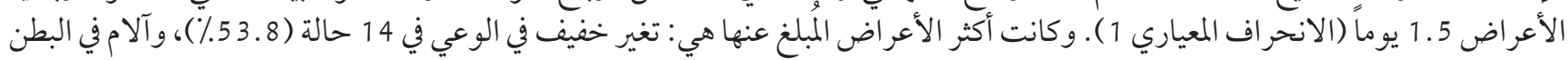

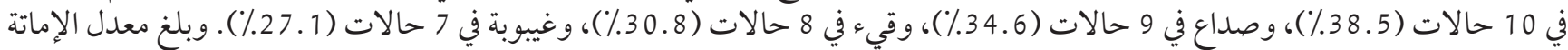

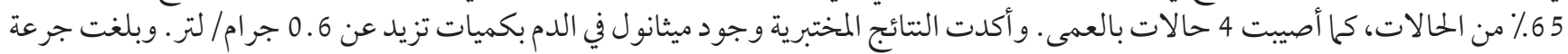

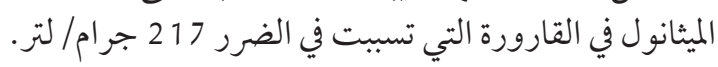

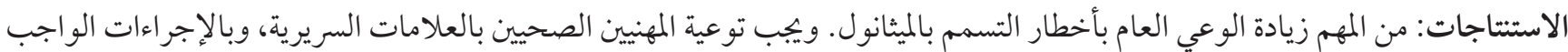
اتخاذها في حالة التسمم بالميثانول.

\section{Références}

1. Methanol. Properties, production, uses, \& poisoning. Encyclopaedia Britannica (s.d). (https://www.britannica.com/science/ methanol, consulté le 20 mars 2020).

2. Zobnine IV, Liubimov BM, Malyh AF, TretyakovAB, Teterina IP, Pazukov EA, et al. Intoxication collective par le méthanol à Irkoutsk en décembre 2016. Toxicol Anal Clin. 2017 May;29(2S):S77-S78. https://doi.org/10.1016/j.toxac.2017.03.118

3. Liu JJ, Daya MR, Carrasquillo O, Kales NS. Prognostic factors in patients with methanol poisoning. J Toxico Clin Toxicol. 1998;36(3):175-81. https://doi.org/10.3109/15563659809028937 
4. Jacobsen D, Jansen H, Wiik $\square$ Larsen E, Bredesen JE, Halvorsen S. Studies on methanol poisoning, Acta Med Scand. 1982;212(12):5-10. https://doi.org/10.1111/j.0954-6820.1982.tb03160.x

5. Palatnick W, Redman LW, Sitar DS, Tenenbein M. Methanol half-life during ethanol administration: implications for management of methanol poisoning. Ann Emerg Med. 1995 Aug;26(2):202-7. https://doi.org/10.1016/S0196-0644(95)70152-4

6. McCoy HG, Cipolle RJ, Ehlers SM, Sawchuk RJ, Zaske DE. Severe methanol poisoning: application of a pharmacokinetic model for ethanol therapy and hemodialysis. Am J Med. 1979 Nov;67(5):804-7. https://doi.org/10.1016/0002-9343(79)90738-1

7. Recensement général de la population et de l'habitat 2014 : Population légale du Maroc. Rabat: Haut-Commissariat au Plan; 2018 (https://www.hcp.ma/downloads/RGPH-2014_t17441.html, consulté le 23 mars 2020).

8. Rhalem N, Jalal G, Soulaymani R. Intoxication par le méthanol. 1996 (https://docplayer.fr/27698320-Intoxication-par-lemethanol-n-rhalem-gh-jalal-r-soulaymani.html).

9. Sutton TL, Foster RL, Liner SR. Acute methanol ingestion. Pediatr Emerg Care. 2002 Oct;18(5):360-3. https://doi. org/10.1097/00006565-200210000-00009

10. Jacobsen D, McMartin KE. Methanol and ethylene glycol poisonings. Mechanism of toxicity,clinical course, diagnosis and treatment. Med Toxicol. 1986 Sep-Oct;1(5):309-34. https://doi.org/10.1007/bf03259846

11. Treichel JL, Henry MM, Skumatz CM, Eells JT, Burke JM. Formate, the toxic metabolite of methanol, in cultured ocular cells. Neurotoxicology. 2003 Dec;24(6):825-34. https://doi.org/10.1016/S0161-813X(03)00059-7

12. Kurtas O, Imre KY, Ozer E, Can M, Birincioglu I, Butun C, et al. The evaluation of deaths due to methyl alcohol intoxication. Biomed Res. 2017;28(8):3680-7.

13. Théfenne H, Turc J, Carmoi T, Gardet V, Renard C. Intoxication aiguë au méthanol : réflexion à partir d'un cas. Ann Biol Clin. 2005;63(5):556-60. (https://docplayer.fr/57683568-Abc-intoxication-aigue-au-methanol-reflexion-a-partir-d-un-cas-pratiquequotidienne.html).

14. United Nations Environment Programme (UNEP)/International Labour Organization (ILO)/World Health Organization (WHO). International Programme on Chemical Safety (IPCS). Methanol. Geneva: World Health Organization; 1997 (Environmental Health Criteria Series, No 196).

15. Paasma R, Hovda KE, Tikkerberi A, Jacobsen D. Methanol mass poisoning in Estonia: outbreak in 154 patients. Clin Toxicol(Phila). 2007;45(2):152-7. https://doi.org/10.1080/15563650600956329.

16. H. Sanaei-Zadeh H, S.K. Esfeh SK, N. Zamani N, F. Jamshidi F, S. Shadnia S. Hyperglycemia is a strong prognostic factor of lethality in methanol poisoning, J Med Toxicol. 2011 Sep;7(3):189-4. https://doi.org/10.1007/s13181-011-0142-x.

17. Francis ST, Nair JR, Shiji PV, Mohamed S, Geetha P, Sasidharan PK. A case series of acute methanol poisoning from Northern Kerala. Emergency Med. 2016;6(2): 312. https://doi.org/10.4172/2165-7548.1000312. 